\title{
Healthcare resource utilization in patients on lipid-lowering therapies outside Western Europe and North America: findings of the cross-sectional observational International ChoLesterol management Practice Study (ICLPS)
}

Lieven Annemans ${ }^{1 *}$, Joseph Azuri ${ }^{2}$, Khalid Al-Rasadi ${ }^{3}$, Ibrahim Al-Zakwani ${ }^{3}$, Veronique Daclin ${ }^{4}$, Florence Mercier ${ }^{5}$ and Nicolas Danchin ${ }^{6}$

\begin{abstract}
Background: Few recent large-scale studies have examined healthcare consumption associated with dyslipidemia in countries outside Western Europe and North America.

Methods: This analysis, from a cross-sectional observational study conducted in 18 countries in Eastern Europe, Asia, Africa, the Middle East and Latin America, evaluated avoidable healthcare consumption (defined as $\geq 1$ hospitalization for cardiovascular reasons or $\geq 1$ visit to the emergency room for any reason in the previous 12 months) in patients receiving stable lipid-lowering therapy (LLT). A total of 9049 patients (aged $\geq 18$ years) receiving LLT for $\geq 3$ months and who had had their low-density lipoprotein cholesterol (LDL-C) value measured on stable LLT in the previous 12 months were enrolled between August 2015 and August 2016. Patients who had received a proprotein convertase subtilisin/kexin type 9 inhibitor in the previous 6 months were excluded. Patients were stratified by cardiovascular risk level using the Systematic Coronary Risk Estimation chart for high-risk countries.

* Correspondence: lieven.annemans@ugent.be

'Department of Public Health, Ghent University, Corneel Heymanslaan 10, 9000 Ghent, Belgium

Full list of author information is available at the end of the article

C C The Author(s). 2020 Open Access This article is licensed under a Creative Commons Attribution 4.0 International License, which permits use, sharing, adaptation, distribution and reproduction in any medium or format, as long as you give appropriate credit to the original author(s) and the source, provide a link to the Creative Commons licence, and indicate if changes were made. The images or other third party material in this article are included in the article's Creative Commons licence, unless indicated otherwise in a credit line to the material. If material is not included in the article's Creative Commons licence and your intended use is not permitted by statutory regulation or exceeds the permitted use, you will need to obtain permission directly from the copyright holder. To view a copy of this licence, visit http://creativecommons.org/licenses/by/4.0/ The Creative Commons Public Domain Dedication waiver (http://creativecommons.org/publicdomain/zero/1.0/) applies to the data made available in this article, unless otherwise stated in a credit line to the data. 
(Continued from previous page)

Results: The proportion of patients at their LDL-C goal was 32.1\% for very-high risk patients compared with 55.7 and $51.9 \%$ for patients at moderate and high cardiovascular risk, respectively. Overall, $20.1 \%$ of patients had $\geq 1$ reported hospitalization in the previous 12 months (7.9\% for cardiovascular reasons), 35.2\% had $\geq 1$ intensive care unit stay and $13.8 \%$ visited the emergency room. Avoidable healthcare resource consumption was reported for $18.7 \%$ patients overall, and in 27.8, 7.7, 7.7 and 13.2\% of patients at very-high, high, moderate and low risk, respectively. Across all risk groups $22.4 \%$ of patients not at LDL-C goal and $16.6 \%$ of patients at LDL-C goal had avoidable healthcare resource consumption.

Being at very-high cardiovascular risk, having cardiovascular risk factors (including hypertension and smoking), and having factors indicating that the patient may be difficult to treat (including statin intolerance, comorbidities and chronic medication), were independent risk factors for avoidable healthcare resource consumption (all $p<0.05)$.

Conclusions: Healthcare resource consumption associated with adverse clinical outcomes was observed in patients on stable LLT in countries outside Western Europe and North America, particularly those at very-high cardiovascular risk and those who were difficult to treat.

Keywords: Dyslipidemia, Health resource, Disease management, Statins, Observational study

\section{Introduction}

Dyslipidemia is a major risk factor for cardiovascular disease, which is a leading cause of mortality and morbidity worldwide [1-3]. Reducing low-density lipoprotein cholesterol (LDL-C) towards recommended treatment goals is a cornerstone of dyslipidemia therapy, and lowers the risk of cardiovascular events $[1,4]$. The aim of healthcare systems internationally is to deliver quality clinical care, including improvements in LDL-C management and associated clinical outcomes. However, these systems also need to be sustainable, and it is important to consider the cost-effectiveness of care, particularly in countries where healthcare resources may be limited.

Dyslipidemia is associated with increased healthcare resource utilization and costs [5-8]. This includes 'desired' healthcare resource consumption, eg, that associated with patient follow-up, which aims to improve patient outcomes, and 'avoidable' consumption of healthcare resources, resulting from clinical complications of dyslipidemia. 'Avoidable' consumption may be higher in patients whose management is suboptimal, ie, difficult-to-treat patients $[9,10]$. Recent large-scale studies of achievement of LDL-C goals in patients with dyslipidemia have been conducted largely in Western Europe and North America [11-13], and data from other parts of the world are limited [14-17]. The International ChoLesterol management Practice Study (ICLPS) was a multinational, crosssectional, observational study to investigate the achievement of European Society of Cardiology (ESC)/European Atherosclerosis Society (EAS) guideline LDL-C goals [1], and their determinants, in real-world clinical practice in countries outside of Western Europe and North America [18]. The primary outcome was the proportion of patients who failed to achieve their LDL-C goal according to the 2011 ESC/EAS guidelines [19]. The results of this study suggested that rates of LDL-C goal achievement were poor in some countries outside Western Europe and North America. This paper will describe healthcare resource consumption in ICLPS, one of the predefined secondary objectives of the study. The findings will add to our understanding of the impact of dyslipidemia on healthcare utilization in countries, for which data are lacking. We hypothesize that avoidable consumption of healthcare resources will be elevated in patients not at LDL-C goal.

\section{Methods}

ICLPS was a multinational, cross-sectional, observational study conducted in 452 centers across 18 countries in Africa, Asia, Eastern Europe, Latin America and the Middle East between August 2015 and August 2016. The methods are described in detail elsewhere [18]. Achievement of LDL-C target was defined according to the $2011 \mathrm{ESC} / \mathrm{EAS}$ guidelines [19], ie, $<1.8 \mathrm{mmol} / \mathrm{L}$ or $50 \%$ LDL-C reduction (for those patients whose baseline untreated LDL-C was available) when target levels could not be reached for very-high risk patients, $<2.5 \mathrm{mmol} / \mathrm{L}$ or $50 \%$ LDL-C reduction for high-risk patients, and $<3.0$ $\mathrm{mmol} / \mathrm{L}$ for moderate-risk patients. A full list of participating physician investigators is provided in the Additional file 1 . The study was conducted according to the Declaration of Helsinki principles, guidelines for Good Epidemiology Practice and local regulations.

\section{Patients}

Patients aged $\geq 18$ years, who had been receiving a stable dose and type of lipid-lowering therapy (LLT) for $\geq 3$ months before enrolment and had had their LDL-C value measured on stable LLT in the previous 12 months, were eligible. Proprotein convertase subtilisin/ kexin type 9 (PCSK9) inhibitors were not available in all countries at the time of the study; therefore, patients who had received a PCSK9 inhibitor in the previous 6 
months were excluded. All consecutive presenting patients, attending their physician for any reason, were enrolled over a predefined 2-week interval at any one site, and within a 3-6-month timeframe for each country.

Patients were stratified according to cardiovascular risk (very-high, high, moderate or low), calculated retrospectively in patients in whom the relevant data were available, using the Systematic Coronary Risk Estimation (SCORE) chart [1]. The chart for high-risk countries was selected for all participating countries as recommended in the European guidelines [1] because of the increasing rate of cardiovascular disease in non-European countries.

\section{Socioeconomic and healthcare consumption variables}

Data on baseline demographics, residence, education, employment status, health insurance and healthcare resource consumption during the previous 12 months were collected on the patient case-report form, which was completed for each patient during a single visit. Healthcare resource consumption variables included the number of, and main reasons for, hospitalizations and visits to emergency rooms (ERs), general practitioners (GPs) and specialists (cardiovascular and other), as well as any investigations carried out (electrocardiogram [ECG], echocardiography, stress ECG and laboratory tests, including tests for cholesterol level).

\section{Statistical analysis}

The presence of avoidable healthcare resource consumption was determined for each patient. Avoidable healthcare resource consumption was defined as at least one hospitalization for a cardiovascular reason or at least one visit to the ER. Healthcare resource consumption variables and avoidable healthcare resource consumption in the past 12 months are presented as descriptive statistics with mean \pm standard deviation or median (interquartile range) for continuous variables, and as counts (percentages) for categorical data.

The factors associated with avoidable healthcare consumption were studied using two mixed categorical models: one including variables at the patient level only, and the other including variables at the patient level as well as environmental variables. Variables with a $p$-value $\leq 10 \%$ in univariate statistics and recorded in more than $80 \%$ of patients were checked for collinearity (with Cramer's V statistics) and reviewed by a scientific expert (ND). Selected variables were finally proposed to the model in a descending stepwise procedure. Variables significant in the multivariable logistic regression analysis at the $5 \%$ level were retained in the final model. $C$-statistics (a measure of goodness of fit of the logistic models) were provided with the respective $95 \%$ confidence intervals. Variables tested but not significant in the multivariable logistic regression analysis at the $5 \%$ level are listed in Additional file 1: Table S1.

All authors had full access to the data presented here, and FM had access to all of the data in the study and performed the data analysis. All authors take responsibility for the study's integrity and data accuracy.

\section{Results}

\section{Patients}

The demographic and socioeconomic profile of the patients is shown in Table 1. A total of 9049 patients were enrolled in the study: 9.7\% from Africa, 39.2\% from Asia, 9.3\% from Eastern Europe, 20.8\% from Latin America and 20.9\% from the Middle East [18]. The mean age of the population was $60.2 \pm 11.7$ years and $55.0 \%$ were male (Table 1); clinically defined (by the investigating physician) hypertension was present in $71.5 \%$ and diabetes mellitus in $54.3 \%$. SCORE cardiovascular risk could be calculated for $87.8 \%$ patients. Of these, $60.9 \%$ were at very-high risk, $33.0 \%$ were at high risk, $5.2 \%$ were at moderate risk and $0.9 \%$ were at low risk of cardiovascular disease.

Most patients (79.8\%) were from urban areas, and $72.7 \%$ had completed secondary education or higher. Overall, $34.8 \%$ of patients worked full-time, $5.0 \%$ worked part-time and $60.2 \%$ of patients were not employed or had retired. The majority of patients (69.1\%) had healthcare insurance: $48.5 \%$ had healthcare covered by national health/public health insurance, $16.5 \%$ had private healthcare and $4.1 \%$ had both. Of the remaining patients, $24.4 \%$ had no insurance coverage and insurance status was unknown in $6.4 \%$. Of patients with health insurance, drug reimbursement was included for $67.8 \%$, which equates to $46.9 \%$ of all patients.

\section{LDL-C goal achievement}

LDL-C goal achievement rates were 55.7, 51.9, and $32.1 \%$ for patients at moderate, high, and very-high cardiovascular risk, respectively [18].

\section{Healthcare resource consumption in the 12 months before enrolment}

Table 2 and Additional file 1: Table S2 present the frequency and duration of hospitalizations in the 12 months before enrolment for all patients, by risk level and by whether or not the LDL-C goal was achieved. Overall, $20.1 \%$ of patients had at least one reported hospitalization in the 12 months before enrolment. In patients with at least one reported hospitalization, the mean hospital stay was $8.0 \pm 11.3$ days per hospitalization during their three most recent hospitalizations. Approximately one-third of patients (35.2\%) with hospitalizations had at least one hospitalization in an intensive care unit among the previous three hospitalizations. Of patients with hospitalizations, $7.9 \%$ had at least one for 
Table 1 Demographic and socioeconomic profile of patients by risk level

\begin{tabular}{|c|c|c|c|c|c|c|}
\hline & $\begin{array}{l}\text { Low Risk } \\
(n=70)\end{array}$ & $\begin{array}{l}\text { Moderate Risk } \\
(n=411)\end{array}$ & $\begin{array}{l}\text { High Risk } \\
(n=2621)\end{array}$ & $\begin{array}{l}\text { Very-High Risk } \\
(n=4842)\end{array}$ & $\begin{array}{l}\text { Risk Non-Assessable } \\
(n=1105)\end{array}$ & $\begin{array}{l}\text { All } \\
(N=9049)\end{array}$ \\
\hline Mean (SD) age, years & $37.6(5.7)$ & $51.8(10.3)$ & $57.5(11.3)$ & $63.3(10.7)$ & $57.6(12.4)$ & $60.2(11.7)$ \\
\hline Male & $8(11.4)$ & $171(41.6)$ & $1152(44.0)$ & $3136(64.8)$ & $508(46.0)$ & $4975(55.0)$ \\
\hline Ethnicity & $n=70$ & $n=411$ & $n=2621$ & $n=4842$ & $n=1105$ & $n=9049$ \\
\hline Asian/South Asian/Indian & $34(48.6)$ & $163(39.7)$ & $1442(55.0)$ & $1765(36.5)$ & $515(46.6)$ & 3919 (43.3) \\
\hline Black African & $1(1.4)$ & $2(0.5)$ & $52(2.0)$ & $47(1.0)$ & $20(1.8)$ & $122(1.3)$ \\
\hline Caucasian/European & $14(20.0)$ & $109(26.5)$ & $490(18.7)$ & $1829(37.8)$ & $232(21.0)$ & $2674(29.6)$ \\
\hline Native Latin American & $5(7.1)$ & $34(8.3)$ & $256(9.8)$ & $357(7.4)$ & $144(13.0)$ & $796(8.8)$ \\
\hline Oriental/Arab/Persian & $6(8.6)$ & $41(10.0)$ & $157(6.0)$ & $490(10.1)$ & $62(5.6)$ & $756(8.4)$ \\
\hline Other & $10(14.3)$ & $62(15.1)$ & $224(8.5)$ & $354(7.3)$ & $132(11.9)$ & $782(8.6)$ \\
\hline Geographical region & $n=70$ & $n=411$ & $n=2621$ & $n=4842$ & $n=1105$ & $n=9049$ \\
\hline Africa & $1(1.4)$ & $20(4.9)$ & $266(10.1)$ & $504(10.4)$ & $90(8.1)$ & $881(9.7)$ \\
\hline Asia & $23(32.9)$ & $135(32.8)$ & $1335(50.9)$ & $1581(32.7)$ & $472(42.7)$ & $3546(39.2)$ \\
\hline Eastern Europe & $2(2.9)$ & $27(6.6)$ & $66(2.5)$ & $699(14.4)$ & $52(4.7)$ & $846(9.3)$ \\
\hline Latin America & $19(27.1)$ & $140(34.1)$ & $554(21.1)$ & $837(17.3)$ & $336(30.4)$ & $1886(20.8)$ \\
\hline Middle East & $25(35.7)$ & $89(21.7)$ & $400(15.3)$ & $1221(25.2)$ & $155(14.0)$ & $1890(20.9)$ \\
\hline Residence location & $n=70$ & $n=411$ & $n=2621$ & $n=4842$ & $n=1105$ & $n=9049$ \\
\hline Urban area & $60(85.7)$ & $351(85.4)$ & $2126(81.1)$ & $3779(78.0)$ & $908(82.2)$ & $7224(79.8)$ \\
\hline Rural area & $3(4.3)$ & $32(7.8)$ & $252(9.6)$ & $515(10.6)$ & $99(9.0)$ & $901(10.0)$ \\
\hline Suburban area & $7(10.0)$ & $28(6.8)$ & $243(9.3)$ & $548(11.3)$ & $98(8.9)$ & $924(10.2)$ \\
\hline Educational level & $n=70$ & $n=411$ & $n=2619$ & $n=4833$ & $n=1102$ & $n=9035$ \\
\hline Illiterate & $2(2.9)$ & $12(2.9)$ & $114(4.4)$ & $290(6.0)$ & $40(3.6)$ & $458(5.1)$ \\
\hline Primary & $8(11.4)$ & $60(14.6)$ & $613(23.4)$ & $1125(23.3)$ & $200(18.1)$ & $2006(22.2)$ \\
\hline Secondary & $19(27.1)$ & $143(34.8)$ & $958(36.6)$ & $1837(38.0)$ & $379(34.4)$ & $3336(36.9)$ \\
\hline University/Higher & $41(58.6)$ & $196(47.7)$ & $934(35.7)$ & $1581(32.7)$ & $483(43.8)$ & 3235 \\
\hline Health insurance & $n=70$ & $n=411$ & $n=2621$ & $n=4842$ & $n=1105$ & $n=9049$ \\
\hline National health service/ public health insurance & $23(32.9)$ & $165(40.1)$ & $970(37.0)$ & $2781(57.4)$ & $452(40.9)$ & $4391(48.5)$ \\
\hline Private health insurance & $16(22.9)$ & $130(31.6)$ & $457(17.4)$ & $657(13.6)$ & $234(21.2)$ & $1494(16.5)$ \\
\hline $\begin{array}{l}\text { National health insurance/ public health insurance + } \\
\text { private health insurance }\end{array}$ & $2(2.9)$ & $12(2.9)$ & $95(3.6)$ & $240(5.0)$ & $24(2.2)$ & $373(4.1)$ \\
\hline No coverage & 27 (38.6) & $94(22.9)$ & $824(31.4)$ & $958(19.8)$ & $307(27.8)$ & $2210(24.4)$ \\
\hline Unknown & $2(2.9)$ & $10(2.4)$ & $275(10.5)$ & $205(4.2)$ & $88(8.0)$ & $580(6.4)$ \\
\hline Insurance includes drug reimbursement & $\begin{array}{l}n=41 \\
29(70.7)\end{array}$ & $\begin{array}{l}n=307 \\
199(64.8)\end{array}$ & $\begin{array}{l}n=1522 \\
1005(66.0)\end{array}$ & $\begin{array}{l}n=3674 \\
2581(70.3)\end{array}$ & $\begin{array}{l}n=708 \\
426(60.2)\end{array}$ & $\begin{array}{l}n=6252 \\
4240(67.8)\end{array}$ \\
\hline Employment status & $n=70$ & $n=410$ & $n=2620$ & $n=4842$ & $n=1105$ & $n=9047$ \\
\hline Full-time & $42(60.0)$ & $230(56.1)$ & $978(37.3)$ & $1452(30.0)$ & $450(40.7)$ & $3152(34.8)$ \\
\hline Part-time & $10(14.3)$ & $25(6.1)$ & $131(5.0)$ & $230(4.8)$ & $52(4.7)$ & $448(5.0)$ \\
\hline Not employed/retired & $18(25.7)$ & $155(37.8)$ & $1511(57.7)$ & $3160(65.3)$ & $603(54.6)$ & $5447(60.2)$ \\
\hline
\end{tabular}

Data are presented as $\mathrm{n}(\%)$ unless otherwise stated

$n$, number of patients in the sample population; $S D$, standard deviation

cardiovascular reasons: $3.3 \%$ for myocardial infarction, $3.1 \%$ for unstable angina, $0.9 \%$ for ischemic stroke, and $2.3 \%$ for coronary revascularization. The proportion of patients with at least one reported hospitalization was $31.2 \%$ in the very-high risk group and $4.3-8.5 \%$ in the other risk groups. Corresponding values for cardiovascular hospitalizations were $14.2 \%$ and $0-0.6 \%$, for the very-high risk group and other risk groups, respectively (Table 2). Of patients who achieved their LDL-C goal, $18.1 \%$ had at least one hospitalization $(8.0 \%$ for cardiovascular reasons) compared with $24.3 \%$ (9.6\%) for patients not at goal (Table 2). 
Table 2 Frequency and duration of hospitalizations in the 12 months before enrolment by risk level and LDL-C goal achievement

\begin{tabular}{|c|c|c|c|c|c|c|c|c|}
\hline & \multicolumn{5}{|l|}{ Risk Level } & \multicolumn{2}{|c|}{ LDL-C Goal Achieved } & \multirow{2}{*}{$\begin{array}{l}\text { All } \\
(N=9049)\end{array}$} \\
\hline & $\begin{array}{l}\text { Low } \\
\text { Risk } \\
(n=70)\end{array}$ & $\begin{array}{l}\text { Moderate } \\
\text { Risk } \\
(n=411)\end{array}$ & $\begin{array}{l}\text { High Risk } \\
(n=2621)\end{array}$ & $\begin{array}{l}\text { Very-High } \\
\text { Risk } \\
(n=4842)\end{array}$ & $\begin{array}{l}\text { Risk Non- } \\
\text { Assessable } \\
(n=1105)\end{array}$ & $\begin{array}{l}\text { Yes } \\
(n=3140)\end{array}$ & $\begin{array}{l}\text { No } \\
(n=4734)\end{array}$ & \\
\hline $\begin{array}{l}\text { Number (\%) of hospitalizations of the patient } \\
\text { in the last } 12 \text { months }\end{array}$ & $n=70$ & $n=411$ & $n=2620$ & $n=4841$ & $n=1105$ & $n=3138$ & $n=4734$ & $n=9047$ \\
\hline At least one & $3(4.3)$ & $31(7.5)$ & $177(6.8)$ & $1512(31.2)$ & $94(8.5)$ & $569(18.1)$ & $1151(24.3)$ & $1817(20.1)$ \\
\hline None & $62(88.6)$ & $369(89.8)$ & $2280(87.0)$ & $3193(66.0)$ & $939(85.0)$ & $2417(77.0)$ & $3425(72.3)$ & $6843(75.6)$ \\
\hline One & $3(4.3)$ & $27(6.6)$ & $150(5.7)$ & $1085(22.4)$ & $73(6.6)$ & $411(13.1)$ & $851(18.0)$ & $1338(14.8)$ \\
\hline Two & 0 & $3(0.7)$ & $22(0.8)$ & $312(6.4)$ & $19(1.7)$ & $114(3.6)$ & $223(4.7)$ & $356(3.9)$ \\
\hline Three & 0 & $1(0.2)$ & $3(0.1)$ & $81(1.7)$ & $1(<0.1)$ & $33(1.1)$ & $52(1.1)$ & $86(1.0)$ \\
\hline More than three & 0 & 0 & $2(<0.1)$ & $34(0.7)$ & $1(<0.1)$ & $11(0.4)$ & $25(0.5)$ & $37(0.4)$ \\
\hline Unknown & $5(7.1)$ & $11(2.7)$ & $163(6.2)$ & $136(2.8)$ & $72(6.5)$ & $152(4.8)$ & $158(3.3)$ & $387(4.3)$ \\
\hline $\begin{array}{l}\text { At least one hospitalization in intensive care unit } \\
\text { among the last three hospitalizations, } \mathrm{n}(\%)\end{array}$ & $\begin{array}{l}n=3 \\
0\end{array}$ & $\begin{array}{l}n=31 \\
2(6.5)\end{array}$ & $\begin{array}{l}n=177 \\
23(13.0)\end{array}$ & $\begin{array}{l}n=1508 \\
599(39.7)\end{array}$ & $\begin{array}{l}n=94 \\
15(16.0)\end{array}$ & $\begin{array}{l}n=568 \\
228(40.1)\end{array}$ & $\begin{array}{l}n=1148 \\
396(34.5)\end{array}$ & $\begin{array}{l}n=1813 \\
639(35.2)\end{array}$ \\
\hline $\begin{array}{l}\text { At least one hospitalization for } \mathrm{CV} \text { reasons among } \\
\text { the last three hospitalizations, } \mathrm{n}(\%)\end{array}$ & $\begin{array}{l}n=65 \\
0\end{array}$ & $\begin{array}{l}n=400 \\
2(0.5)\end{array}$ & $\begin{array}{l}n=2458 \\
9(0.4)\end{array}$ & $\begin{array}{l}n=4708 \\
667(14.2)\end{array}$ & $\begin{array}{l}n=1033 \\
6(0.6)\end{array}$ & $\begin{array}{l}n=2989 \\
240(8.0)\end{array}$ & $\begin{array}{l}n=4577 \\
438(9.6)\end{array}$ & $\begin{array}{l}n=8664 \\
684(7.9)\end{array}$ \\
\hline For myocardial infarction & 0 & 0 & $1(<0.1)$ & $286(6.1)$ & $2(0.2)$ & $116(3.9)$ & $171(3.7)$ & $289(3.3)$ \\
\hline For unstable angina & 0 & $2(0.5)$ & $7(0.3)$ & $255(5.4)$ & $3(0.3)$ & $85(2.8)$ & $179(3.9)$ & $267(3.1)$ \\
\hline For ischemic stroke & 0 & 0 & $1(<0.1)$ & $76(1.6)$ & $1(<0.1)$ & $21(0.7)$ & $56(1.2)$ & $78(0.9)$ \\
\hline For coronary revascularization & 0 & 0 & $2(<0.1)$ & $196(4.2)$ & $1(<0.1)$ & $70(2.3)$ & $128(2.8)$ & $199(2.3)$ \\
\hline Durations (days) of last three hospitalizations ${ }^{a}$ & $n=3$ & $n=30$ & $n=176$ & $n=1471$ & $n=92$ & $n=557$ & $n=1120$ & $n=1772$ \\
\hline Mean \pm SD & $3.7 \pm 3.1$ & $8.4 \pm 4.5$ & $6.5 \pm 6.2$ & $8.2 \pm 12.1$ & $7.6 \pm 6.3$ & $8.7 \pm 17.6$ & $7.7 \pm 6.8$ & $8.0 \pm 11.3$ \\
\hline Median (IQR) & $\begin{array}{l}3.0 \\
(1.0-7.0)\end{array}$ & $\begin{array}{l}9.5 \\
(4.0-11.0)\end{array}$ & $\begin{array}{l}4.0 \\
(2.0-10.0)\end{array}$ & $\begin{array}{l}6.0 \\
(3.0-10.0)\end{array}$ & $\begin{array}{l}6.0 \\
(2.2-10.5)\end{array}$ & $\begin{array}{l}6.0 \\
(3.0-10.0)\end{array}$ & $\begin{array}{l}6.0 \\
(3.0-10.0)\end{array}$ & $\begin{array}{l}6.0 \\
(3.0-10.0)\end{array}$ \\
\hline \multicolumn{9}{|l|}{ Durations (days) of last three hospitalizations ${ }^{a}$} \\
\hline For myocardial infarction & - & - & $n=1$ & $n=275$ & $n=1$ & $n=112$ & $n=164$ & $n=277$ \\
\hline Mean \pm SD & & & 23.0 & $7.3 \pm 5.6$ & 1.0 & $7.7 \pm 5.5$ & $7.1 \pm 5.9$ & $7.3 \pm 5.7$ \\
\hline Median (IQR) & - & - & $\begin{array}{l}23.0 \\
(23.0-23.0)\end{array}$ & $\begin{array}{l}5.0 \\
(3.0-9.0)\end{array}$ & $\begin{array}{l}1.0 \\
(1.0-1.0)\end{array}$ & $\begin{array}{l}7.0 \\
(3.0-10.0)\end{array}$ & $\begin{array}{l}5.0 \\
(3.5-8.0)\end{array}$ & $\begin{array}{l}5.0 \\
(3.0-9.0)\end{array}$ \\
\hline For unstable angina & - & $n=2.0$ & $n=7$ & $n=254$ & $n=3$ & $n=85$ & $n=178$ & $n=266$ \\
\hline Mean \pm SD & - & $3.0 \pm 0.0$ & $6.7 \pm 7.3$ & $6.4 \pm 4.6$ & $8.3 \pm 5.9$ & $6.1 \pm 4.6$ & $6.5 \pm 4.6$ & $6.4 \pm 4.6$ \\
\hline Median (IQR) & - & $\begin{array}{l}3.0 \\
(3.0-3.0)\end{array}$ & $\begin{array}{l}4.0 \\
(2.0-12.0)\end{array}$ & $\begin{array}{l}5.0 \\
(3.0-10.0)\end{array}$ & $\begin{array}{l}6.0 \\
(4.0-15.0)\end{array}$ & $\begin{array}{l}4.5 \\
(3.0-8.0)\end{array}$ & $\begin{array}{l}5.0 \\
(3.0-10.0)\end{array}$ & $\begin{array}{l}5.0 \\
(3.0-10.0)\end{array}$ \\
\hline For ischemic stroke & - & - & $n=1$ & $n=76$ & $n=1$ & $n=21$ & $n=56$ & $n=78$ \\
\hline Mean \pm SD & - & - & $1.0(0.0)$ & $10.4 \pm 12.9$ & $15.0 \pm 0.0$ & $11.6 \pm 21.4$ & $9.8 \pm 7.8$ & $10.3 \pm 12.8$ \\
\hline Median (IQR) & - & - & $\begin{array}{l}1.0 \\
(1.0-1.0)\end{array}$ & $\begin{array}{l}7.5 \\
(5.0-12.0)\end{array}$ & $\begin{array}{l}15.0 \\
(15.0-15.0)\end{array}$ & $\begin{array}{l}6.0 \\
(4.0-11.0)\end{array}$ & $\begin{array}{l}8.0 \\
(5.0-13.25)\end{array}$ & $\begin{array}{l}7.5 \\
(5.0-12.0)\end{array}$ \\
\hline For coronary revascularization & - & - & $n=2$ & $n=194$ & $n=1$ & $n=70$ & $n=126$ & $n=197$ \\
\hline Mean \pm SD & - & - & $1.5 \pm 0.7$ & $5.6 \pm 5.4$ & 1.0 & $5.9 \pm 6.2$ & $5.4 \pm 4.8$ & $5.6 \pm 5.4$ \\
\hline Median (IQR) & - & - & $\begin{array}{l}1.5 \\
(1.0-2.0)\end{array}$ & $\begin{array}{l}4.0 \\
(2.0-7.0)\end{array}$ & $\begin{array}{l}1.0 \\
(1.0-1.0)\end{array}$ & $\begin{array}{l}3.5 \\
(2.0-8.0)\end{array}$ & $\begin{array}{l}4.0 \\
(2.0-6.0)\end{array}$ & $\begin{array}{l}4.0 \\
(2.0-7.0)\end{array}$ \\
\hline
\end{tabular}

${ }^{\mathrm{a}}$ Mean of last three hospitalizations

$C V$, cardiovascular; $I Q R$, interquartile range; $L D L-C$, low-density lipoprotein cholesterol; $n$, number of patients in the sample population; $S D$, standard deviation

The number of visits to ERs, GPs and specialists, and the number of tests performed in the 12 months before enrolment are shown in Table 3. Patients visited an ER a mean of $0.32 \pm 1.11$ times. The mean number of visits to GPs, cardiovascular specialists and other specialists, was $2.71 \pm 3.87, \quad 1.83 \pm 2.14$ and $2.49 \pm 4.30$, respectively.
Patients underwent a mean of $1.57 \pm 2.16$ ECGs, $0.56 \pm$ 0.81 echocardiography tests and $0.18 \pm 0.45$ stress ECGs. For $98.3 \%$ of the patients, the investigator noted a laboratory test that included cholesterol in the 12 months before enrolment ( $2.10 \pm 1.45$ tests); however, it should be noted that the inclusion criteria of the study required all patients 
Table 3 Number of visits to ERs, GPs and specialists, number of tests, and avoidable healthcare resource consumption in the 12 months before enrolment by risk level and LDL-C goal achievement

\begin{tabular}{|c|c|c|c|c|c|c|c|c|}
\hline & \multicolumn{5}{|l|}{ Risk Level } & \multicolumn{2}{|c|}{ LDL-C Goal Achieved } & \multirow{2}{*}{$\begin{array}{l}\text { All } \\
(N=9049)\end{array}$} \\
\hline & $\begin{array}{l}\text { Low Risk } \\
(n=70)\end{array}$ & $\begin{array}{l}\text { Moderate } \\
\text { Risk } \\
(n=411)\end{array}$ & $\begin{array}{l}\text { High Risk } \\
(n=2621)\end{array}$ & $\begin{array}{l}\text { Very-High } \\
\text { Risk } \\
(n=4842)\end{array}$ & $\begin{array}{l}\text { Risk Non- } \\
\text { Assessable } \\
(n=1105)\end{array}$ & $\begin{array}{l}\text { Yes } \\
(n=3140)\end{array}$ & $\begin{array}{l}\text { No } \\
(n=4734)\end{array}$ & \\
\hline $\begin{array}{l}\text { At least one visit to the ER in the last } 12 \\
\text { months, } \mathrm{n}(\%)\end{array}$ & $\begin{array}{l}n=68 \\
9(13.2)\end{array}$ & $\begin{array}{l}n=395 \\
30(7.6)\end{array}$ & $\begin{array}{l}n=2440 \\
194(8.0)\end{array}$ & $\begin{array}{l}n=4649 \\
865(18.6)\end{array}$ & $\begin{array}{l}n=1053 \\
89(8.5)\end{array}$ & $\begin{array}{l}n=2955 \\
353(11.9)\end{array}$ & $\begin{array}{l}n=4529 \\
736(16.3)\end{array}$ & $\begin{array}{l}n=8605 \\
1187(13.8)\end{array}$ \\
\hline \multicolumn{9}{|l|}{ Number of visits to: } \\
\hline$A n E R$ & $n=52$ & $n=319$ & $n=1863$ & $n=3773$ & $n=820$ & $n=2355$ & $n=3600$ & $n=6827$ \\
\hline Mean \pm SD & $0.23 \pm 0.55$ & $0.21 \pm 0.82$ & $0.19 \pm 0.75$ & $0.42 \pm 1.32$ & $0.19 \pm 0.78$ & $0.26 \pm 0.92$ & $0.39 \pm 1.28$ & $0.32 \pm 1.11$ \\
\hline Median (IQR) & $\begin{array}{l}0.0 \\
(0.0-0.0)\end{array}$ & $\begin{array}{l}0.0 \\
(0.0-0.0)\end{array}$ & $\begin{array}{l}0.0 \\
(0.0-0.0)\end{array}$ & $\begin{array}{l}0.0 \\
(0.0-0.0)\end{array}$ & $\begin{array}{l}0.0 \\
(0.0-0.0)\end{array}$ & $\begin{array}{l}0.0 \\
(0.0-0.0)\end{array}$ & $\begin{array}{l}0.0 \\
(0.0-0.0)\end{array}$ & $\begin{array}{l}0.0 \\
(0.0-0.0)\end{array}$ \\
\hline A GP & $n=57$ & $n=330$ & $n=2017$ & $n=3747$ & $n=902$ & $n=2438$ & $n=3656$ & $n=7053$ \\
\hline Mean \pm SD & $2.54 \pm 2.40$ & $2.78 \pm 3.89$ & $2.91 \pm 3.34$ & $2.66 \pm 4.30$ & $2.44 \pm 3.06$ & $2.66 \pm 3.69$ & $2.81 \pm 4.17$ & $2.71 \pm 3.87$ \\
\hline Median (IQR) & $\begin{array}{l}2.0 \\
(0.0-4.0)\end{array}$ & $\begin{array}{l}2.0 \\
(0.0-4.0)\end{array}$ & $\begin{array}{l}2.0 \\
(0.0-4.0)\end{array}$ & $\begin{array}{l}1.0 \\
(0.0-4.0)\end{array}$ & $\begin{array}{l}2.0 \\
(0.0-3.0)\end{array}$ & $\begin{array}{l}2.0 \\
(0.0-4.0)\end{array}$ & $\begin{array}{l}2.0 \\
(0.0-4.0)\end{array}$ & $\begin{array}{l}2.0 \\
(0.0-4.0)\end{array}$ \\
\hline A CV specialist & $n=55$ & $n=345$ & $n=1992$ & $n=4402$ & $n=870$ & $n=2630$ & $n=4109$ & $n=7664$ \\
\hline Mean \pm SD & $1.05 \pm 1.63$ & $1.41 \pm 1.71$ & $0.99 \pm 1.53$ & $2.37 \pm 2.32$ & $1.30 \pm 1.77$ & $1.92 \pm 2.30$ & $1.90 \pm 2.10$ & $1.83 \pm 2.14$ \\
\hline Median (IQR) & $\begin{array}{l}0.0 \\
(0.0-2.0)\end{array}$ & $\begin{array}{l}1.0 \\
(0.0-2.0)\end{array}$ & $\begin{array}{l}0.0 \\
(0.0-2.0)\end{array}$ & $\begin{array}{l}2.0 \\
(1.0-3.0)\end{array}$ & $\begin{array}{l}1.0 \\
(0.0-2.0)\end{array}$ & $\begin{array}{l}1.0 \\
(0.0-3.0)\end{array}$ & $\begin{array}{l}2.0 \\
(0.0-3.0)\end{array}$ & $\begin{array}{l}1.0 \\
(0.0-3.0)\end{array}$ \\
\hline Another specialist & $n=58$ & $n=337$ & $n=1991$ & $n=3845$ & $n=800$ & $n=2441$ & $n=3732$ & $n=7031$ \\
\hline Mean \pm SD & $2.81 \pm 4.59$ & $2.12 \pm 3.78$ & $2.15 \pm 3.47$ & $2.91 \pm 4.93$ & $1.38 \pm 2.44$ & $2.74 \pm 4.72$ & $2.55 \pm 4.29$ & $2.49 \pm 4.30$ \\
\hline Median (IQR) & $\begin{array}{l}2.0 \\
(0.0-3.0)\end{array}$ & $\begin{array}{l}1.0 \\
(0.0-3.0)\end{array}$ & $\begin{array}{l}1.0 \\
(0.0-3.0)\end{array}$ & $\begin{array}{l}1.0 \\
(0.0-4.0)\end{array}$ & $\begin{array}{l}0.0 \\
(0.0-2.0)\end{array}$ & $\begin{array}{l}1.0 \\
(0.0-3.0)\end{array}$ & $\begin{array}{l}1.0 \\
(0.0-3.0)\end{array}$ & $\begin{array}{l}1.0 \\
(0.0-3.0)\end{array}$ \\
\hline \multicolumn{9}{|l|}{ Number of tests } \\
\hline ECG & $n=62$ & $n=352$ & $n=2125$ & $n=4379$ & $n=927$ & $n=2678$ & $n=4178$ & $n=7845$ \\
\hline Mean \pm SD & $0.92 \pm 0.84$ & $0.88 \pm 0.95$ & $1.08 \pm 1.21$ & $1.98 \pm 2.62$ & $1.08 \pm 1.15$ & $1.58 \pm 2.52$ & $1.69 \pm 2.07$ & $1.57 \pm 2.16$ \\
\hline Median (IQR) & $\begin{array}{l}1.0 \\
(0.0-1.0)\end{array}$ & $\begin{array}{l}1.0 \\
(0.0-1.0)\end{array}$ & $\begin{array}{l}1.0 \\
(0.0-1.0)\end{array}$ & $\begin{array}{l}1.0 \\
(0.0-3.0)\end{array}$ & $\begin{array}{l}1.0 \\
(0.0-1.0)\end{array}$ & $\begin{array}{l}1.0 \\
(0.0-2.0)\end{array}$ & $\begin{array}{l}1.0 \\
(1.0-2.0)\end{array}$ & $\begin{array}{l}1.0 \\
(0.0-2.0)\end{array}$ \\
\hline Echocardiography & $n=55$ & $n=334$ & $n=1949$ & $n=4218$ & $n=848$ & $n=2549$ & $n=3952$ & $n=7404$ \\
\hline Mean \pm SD & $0.29 \pm 0.46$ & $0.34 \pm 0.53$ & $0.29 \pm 0.56$ & $0.75 \pm 0.91$ & $0.34 \pm 0.60$ & $0.53 \pm 0.79$ & $0.63 \pm 0.85$ & $0.56 \pm 0.81$ \\
\hline Median (IQR) & $\begin{array}{l}0.0 \\
(0.0-1.0)\end{array}$ & $\begin{array}{l}0.0 \\
(0.0-1.0)\end{array}$ & $\begin{array}{l}0.0 \\
(0.0-0.0)\end{array}$ & $\begin{array}{l}1.0 \\
(0.0-1.0)\end{array}$ & $\begin{array}{l}0.0 \\
(0.0-1.0)\end{array}$ & $\begin{array}{l}0.0 \\
(0.0-1.0)\end{array}$ & $\begin{array}{l}0.0 \\
(0.0-1.0)\end{array}$ & $\begin{array}{l}0.0 \\
(0.0-1.0)\end{array}$ \\
\hline Stress ECG & $n=54$ & $n=323$ & $n=1914$ & $n=3857$ & $n=827$ & $n=2396$ & $n=3698$ & $n=6975$ \\
\hline Mean \pm SD & $0.15 \pm 0.36$ & $0.18 \pm 0.40$ & $0.10 \pm 0.32$ & $0.22 \pm 0.51$ & $0.15 \pm 0.39$ & $0.17 \pm 0.44$ & $0.19 \pm 0.47$ & $0.18 \pm 0.45$ \\
\hline Median (IQR) & $\begin{array}{l}0.0 \\
(0.0-0.0)\end{array}$ & $\begin{array}{l}0.0 \\
(0.0-0.0)\end{array}$ & $\begin{array}{l}0.0 \\
(0.0-0.0)\end{array}$ & $\begin{array}{l}0.0 \\
(0.0-0.0)\end{array}$ & $\begin{array}{l}0.0 \\
(0.0-0.0)\end{array}$ & $\begin{array}{l}0.0 \\
(0.0-0.0)\end{array}$ & $\begin{array}{l}0.0 \\
(0.0-0.0)\end{array}$ & $\begin{array}{l}0.0 \\
(0.0-0.0)\end{array}$ \\
\hline Lab test including cholesterol & $n=68$ & $n=403$ & $n=2545$ & $n=4695$ & $n=1062$ & $n=3053$ & $n=4590$ & $n=8773$ \\
\hline Mean \pm SD & $2.41 \pm 1.35$ & $2.26 \pm 1.18$ & $2.06 \pm 1.29$ & $2.16 \pm 1.62$ & $1.86 \pm 1.03$ & $2.13 \pm 1.58$ & $2.14 \pm 1.44$ & $2.10 \pm 1.45$ \\
\hline Median (IQR) & $\begin{array}{l}2.0 \\
(2.0-3.0)\end{array}$ & $\begin{array}{l}2.0 \\
(1.0-3.0)\end{array}$ & $\begin{array}{l}2.0 \\
(1.0-2.0)\end{array}$ & $\begin{array}{l}2.0 \\
(1.0-3.0)\end{array}$ & $\begin{array}{l}2.0 \\
(1.0-2.0)\end{array}$ & $\begin{array}{l}2.0 \\
(1.0-3.0)\end{array}$ & $\begin{array}{l}2.0 \\
(1.0-3.0)\end{array}$ & $\begin{array}{l}2.0 \\
(1.0-3.0)\end{array}$ \\
\hline Avoidable healthcare consumption ${ }^{\mathrm{a}}, n(\%)$ & $\begin{array}{l}n=68 \\
9(13.2)\end{array}$ & $\begin{array}{l}n=405 \\
31(7.7)\end{array}$ & $\begin{array}{l}n=2584 \\
200(7.7)\end{array}$ & $\begin{array}{l}n=4799 \\
1332(27.8)\end{array}$ & $\begin{array}{l}n=1073 \\
94(8.8)\end{array}$ & $\begin{array}{l}n=3099 \\
515(16.6)\end{array}$ & $\begin{array}{l}n=4689 \\
1048(22.4)\end{array}$ & $\begin{array}{l}n=8929 \\
1666(18.7)\end{array}$ \\
\hline
\end{tabular}

${ }^{a}$ Defined as at least one hospitalization for $\mathrm{CV}$ reasons or at least one visit to the $\mathrm{ER}$

$C V$, cardiovascular; $E C G$, electrocardiogram; $E R$, emergency room; $G P$, general practitioner; IQR, interquartile range; $L D L-C$, low-density lipoprotein cholesterol; $n$, number of patients in the sample population; $S D$, standard deviation

to have had at least one LDL-C value measured in the previous 12 months. In the very-high risk group, $18.6 \%$ of patients had at least one visit to an ER compared with 7.6$13.2 \%$ of patients in other risk groups (Table 3 ).

\section{Avoidable healthcare resource consumption}

Across all patients, $18.7 \%$ had avoidable healthcare resource consumption in the previous 12 months (Table 3). This was $27.8 \%$ in patients in the very-high risk 
group compared with $7.7-13.2 \%$ in patients in other risk groups, and $22.4 \%$ in patients not at LDL-C goal compared with $16.6 \%$ in patients at LDL-C goal.

The findings of the multivariable analysis of avoidable healthcare resource consumption based on variables at the patient level only are shown in Fig. 1. Younger age, hypertension, congestive heart failure, chronic medication, documented cardiovascular disease, family history of cardiovascular disease, history of hypoglycemia, neurocognitive disorders, smoking, being on the highest tolerated dose of statin and statin intolerance were all independently associated with avoidable healthcare resource consumption. Moderate and high versus veryhigh cardiovascular risk, being educated, full-time employment and being physically active were associated with decreased avoidable healthcare resource consumption. When the multivariable analysis was performed using variables at the patient level and environmental variables (Fig. 2), the following additional independent risk factors for avoidable healthcare resource consumption were identified: the enrolling physician was in a public hospital, the patient had increased heart rate, the practice treated private (mostly private or mixed public and private) patients, the enrolling physician was a cardiologist and the patient had intolerance to two statins.
Additional factors associated with a decrease in avoidable healthcare resource consumption were an investigator's assessment (based on their clinical judgement) of patient's risk as low, the practice was seeing $>25$ patients per day, and the physician having a specialty of 'other'.

The proportion of patients with avoidable healthcare resource consumption differed by type of health insurance (none, 14\%; public, 22.6\%; private, 16.3\%; both public and private, $16.3 \% ; p \leq 0.001$ ) when tested using univariate statistics. However, this effect was not retained in the multivariate models (Additional file 1: Table S1).

\section{Discussion}

In this multinational, cross-sectional, observational study, avoidable healthcare resource consumption was observed in $18.7 \%$ of patients on stable LLT in countries outside Western Europe and North America (16.6\% of patients at LDL-C goal; $22.4 \%$ of patients not at goal), reflecting a high rate of adverse clinical outcomes among this population. Avoidable healthcare resource consumption occurred in $27.8 \%$ of patients at very-high cardiovascular risk, at least double the proportion of patients at lower risk. LDL-C goal achievement rate in the very-high risk group was particularly low $(32.1 \%$ vs 55.7 and $51.9 \%$ for patients at moderate and high risk,

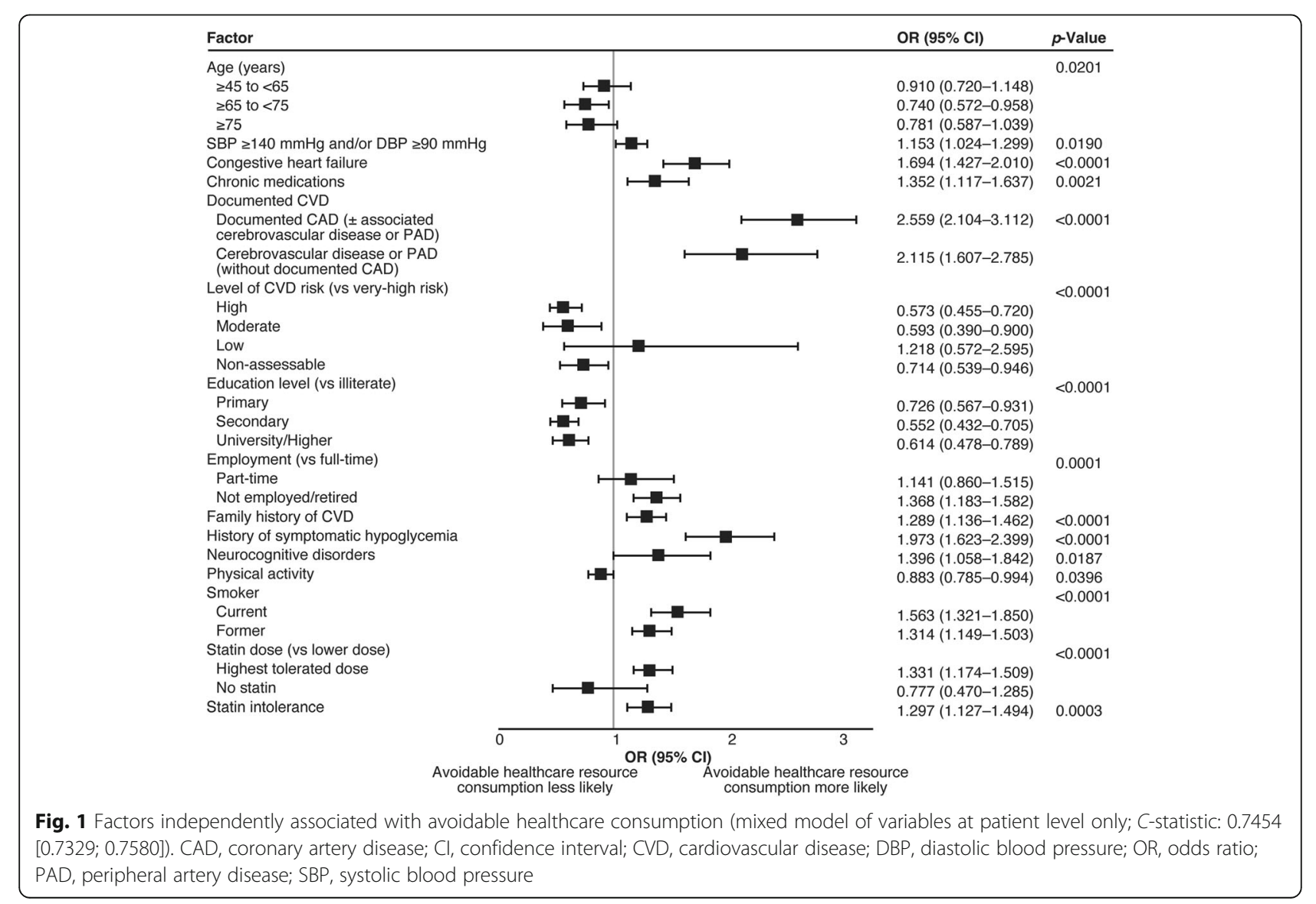




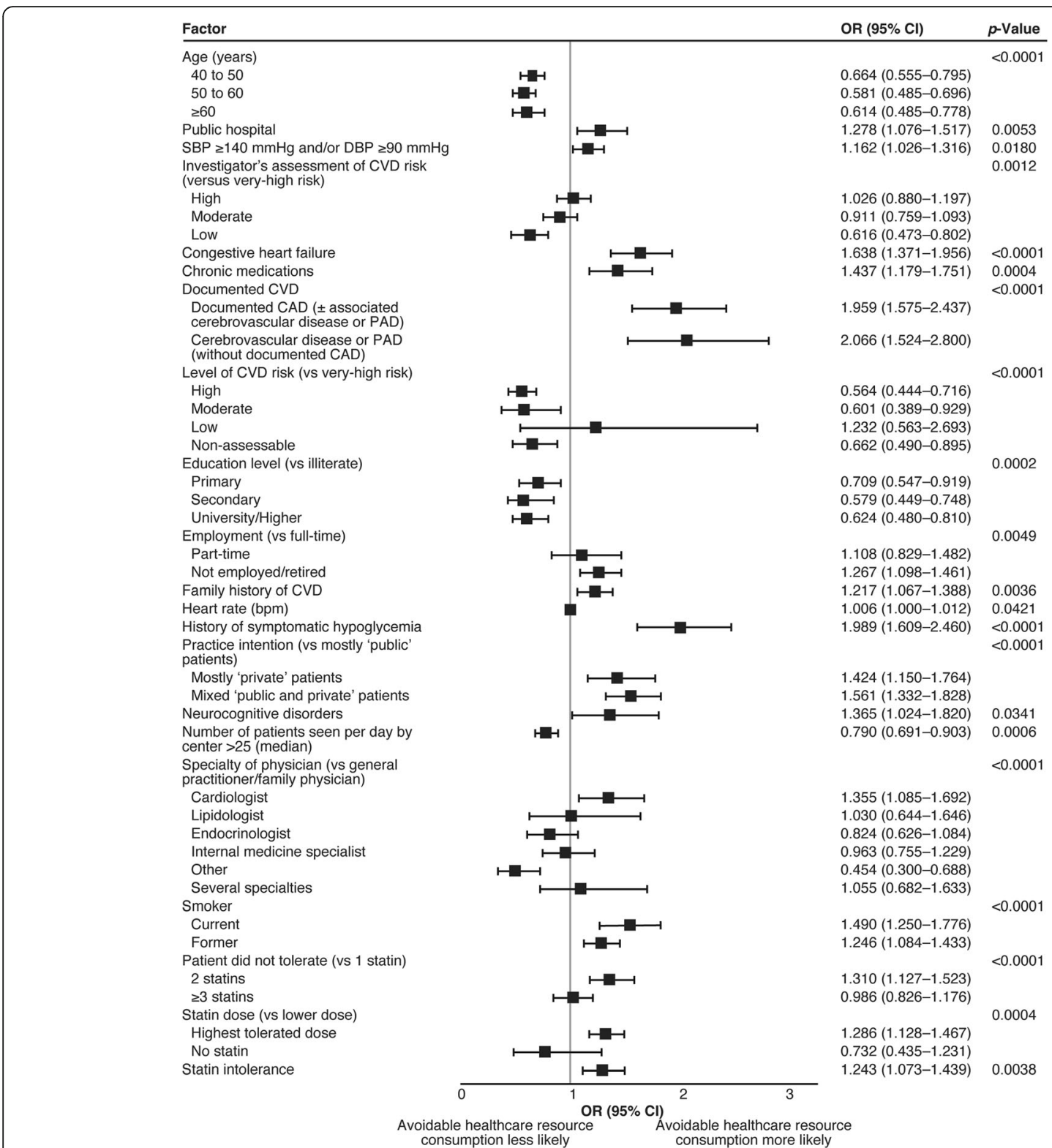

Fig. 2 Factors independently associated with avoidable consumption of healthcare resources (mixed model of variables at patient level and environmental variables; C-statistic: 0.7591 [0.7466; 0.7715]). CAD, coronary artery disease; Cl, confidence interval; CVD, cardiovascular disease; DBP, diastolic blood pressure; OR, odds ratio; PAD, peripheral artery disease; SBP, systolic blood pressure

respectively). Factors that may contribute to this low LDL-C goal achievement rate in very-high risk patients include suboptimal use of LLTs [18], more stringent LDL-C goals (ie, $<70 \mathrm{mg} / \mathrm{dL}$ ) and either failure of physicians to adhere to them in this group or a lack of efficacy of currently used LLTs for achieving these goals. Previous studies in Europe and the USA have reported an increase in healthcare resource utilization associated with cardiovascular outcomes in patients with dyslipidemia that persists for several years after an acute cardiovascular event $[6,7]$. This healthcare burden may be decreased by effective management of LDL-C levels to prevent cardiovascular events [8].

The findings of the multivariable analyses suggested several factors independently associated with avoidable healthcare resource consumption in this patient population. Avoidable healthcare resource consumption was as least twice as high in patients with than without documented cardiovascular disease. It was also higher in patients with other cardiovascular risk factors, including elevated blood pressure, smoking, history of symptomatic hypoglycemia and family history of cardiovascular disease. 
The presence of congestive heart failure, neurocognitive disorders, the use of chronic medications, taking the highest tolerated dose of statin or being statin intolerant were also associated with higher avoidable healthcare resource consumption. Although we cannot draw a conclusion on a formal causal relationship because of the study design, these data may reflect a higher rate of complications in difficult-to-treat patients, ie, those who cannot tolerate effective doses of statins, whose dyslipidemia is resistant to statins, and whose physicians may be reluctant to treat aggressively due to the presence of comorbidities and/or the use of concomitant therapies. These findings are consistent with the results of other studies that have investigated healthcare resource consumption in difficult-to-treat patients. For example, a recent US observational study of data from an integrated health system's electronic health records between 2008 and 2014 compared outcomes between statin-intolerant patients and matched controls (statin-treated patients matched for age, sex, cardiovascular risk category, comorbidities and concomitant medications using propensity score matching) [9]. Patients with statin intolerance were less likely to reach LDL-C goals, incurred higher healthcare costs and experienced a higher rate of adverse cardiovascular events compared with controls. Another retrospective cohort study demonstrated a significantly higher frequency of visits to the ER, and higher total healthcare costs, in patients with low adherence to statin therapy (defined as medication possession ratio $<40 \%$ ) over a 1 -year follow-up period after treatment initiation [10].

Patient factors associated with lower avoidable healthcare resource consumption, such as being educated, in full-time employment and physically active, may indicate a greater ability or commitment of the patient to managing their dyslipidemia. The influence of physician factors on avoidable healthcare resource consumption likely results from variations in the severity of patients seen by different specialties of physicians, the practice's level of experience with dyslipidemia, and differences in the availability of resources between the public and private healthcare sectors. Low cardiovascular risk assessed by SCORE was associated with higher avoidable healthcare resource consumption, whereas low cardiovascular risk assessed by the investigator was associated with lower avoidable healthcare resource consumption. This difference may reflect the low agreement between SCORE and investigator-assessed risk level in the study [18], and highlights the importance of accurate risk assessment to optimize LDL-C management and improve clinical outcomes.

\section{Limitations}

This study was subject to limitations that may have influenced its findings; these have been outlined previously
[18]. Of note here is the cross-sectional nature of the study, which does not allow investigation of causal effects. Furthermore, the study population is not fully representative of all patients treated with LLT in each country. The patients were mainly educated urban residents with health insurance, and as a result were likely to have better access to healthcare than the general population. Consequently, the results may overestimate both LDL-C goal achievement and healthcare resource consumption in these countries. The study population is heterogenous being enrolled from countries with different healthcare systems and cardiovascular risks. Africa and Eastern Europe were underrepresented in the study population. In addition, recording of healthcare resource consumption data by investigators was not always complete; therefore, missing data may also have influenced the interpretation of results. As avoidable healthcare resource consumption was defined as $\geq 1$ hospitalization for cardiovascular reasons or $\geq 1$ visit to the emergency room for any reason in the previous 12 months, it may have included some healthcare resource consumption related to causes other than dyslipidemia. Finally, as healthcare resource consumption was studied in the year preceding study entry but the duration of LLT varied between patients (inclusion criterion: $>3$ months prior to enrolment) it cannot be ruled out that the clinical events leading to increased healthcare resource consumption may have influenced achievement of LDL-C goals thereafter. It would be likely, however, that events such as acute coronary syndromes would have led to optimization of LLT, rather than the reverse.

\section{Conclusions}

Avoidable healthcare resource consumption was observed in approximately one-fifth of patients on stable LLT in countries outside Western Europe and North America, and in more than one-quarter of patients at very-high cardiovascular risk. Being at very-high cardiovascular risk, having cardiovascular risk factors and having factors indicating difficult-to-treat patients, were independently associated with an increased avoidable healthcare consumption.

\section{Supplementary information}

Supplementary information accompanies this paper at https://doi.org/10. 1186/s12944-020-01235-5.

\section{Additional file 1:.}

\section{Abbreviations}

CAD: Coronary artery disease; Cl: Confidence interval; CV: Cardiovascular; CVD: cardiovascular disease; DBP: Diastolic blood pressure; ECG: Electrocardiogram; ESC: European Society of Cardiology; EAS: European Atherosclerosis Society; ER: Emergency room; GP: General practitioner; ICLPS: International ChoLesterol management Practice Study:

IQR: Interquartile range; LDL-C: Low-density lipoprotein cholesterol; 
LLT: Lipid-lowering therapy; n: Number of patients in the sample population; OR: Odds ratio; PAD: Peripheral artery disease; PCSK9: Proprotein convertase subtilisin/kexin type 9; SBP: Systolic blood pressure; SCORE: Systematic Coronary Risk Estimation; SD: Standard deviation

\section{Acknowledgements}

The authors would like to thank the patients, their families, and all investigators involved in this study. Medical writing assistance and editorial support, under the direction of the authors, were provided by Fiona Van, $\mathrm{PhD}$, and Elke Sims, MLangTrans, both of Prime (Knutsford, UK), and funded by Sanofi according to Good Publication Practice guidelines (https:/annals. org/aim/fullarticle/2424869/good-publication-practice-communicatingcompany-sponsored-medical-research-gpp3).

\section{Authors' contributions}

VD and ND contributed to the design of the study. JA and KAR contributed to the acquisition of the data. LA, JA, KAR and IAZ contributed to the interpretation of the data. FM conducted the analysis. All authors critically reviewed the manuscript. All authors meet the criteria for authorship. The corresponding author had full access to all the data and had final responsibility for the decision to submit for publication. The author(s) read and approved the final manuscript.

\section{Funding}

The study was sponsored by Sanofi. Sanofi was involved in the study design, and collection, analysis and interpretation of data, as well as data checking of information provided in the manuscript. However, ultimate responsibility for opinions, conclusions and data interpretation lies with the authors.

\section{Availability of data and materials}

Qualified researchers may request access to patient level data and related study documents including the clinical study report, study protocol with any amendments, blank case report form, statistical analysis plan and dataset specifications. Patient level data will be anonymized and study documents will be redacted to protect the privacy of trial participants. Further details on Sanofi's data sharing criteria, eligible studies and process for requesting access can be found at: https://www.clinicalstudydatarequest.com

\section{Ethics approval and consent to participate}

Local or regional institutional review boards and/or ethics committee approval was obtained, where required. Patients provided written informed consent.

\section{Consent for publication}

Not applicable.

\section{Competing interests}

LA reports non-financial support from Sanofi during the conduct of the study; and personal fees from Sanofi, Amgen and Merck outside the submitted work. JA reports non-financial support from Sanofi during the conduct of the study; and personal fees from Pfizer and non-financial support from Janssen Pharmaceuticals outside the submitted work. KA-R reports non-financial support from Sanofi during the conduct of the study. IA-Z reports nonfinancial support from Sanofi during the conduct of the study. VD reports non-financial support from Sanofi during the conduct of the study; and other support from Sanofi outside the submitted work. FM reports grants from Sanofi and other support from Stat Process during the conduct of the study. ND reports non-financial support from Sanofi during the conduct of the study; and grants, personal fees and non-financial support from Amgen, AstraZeneca and Bristol-Myers Squibb, grants and personal fees from Bayer, Boehringer Ingelheim, Daiichi Sankyo, Eli Lilly, Merck Sharp Dohme, Pfizer and Sanofi, and personal fees from Intercept, Novo Nordisk and Servier outside the submitted work.

\section{Author details}

'Department of Public Health, Ghent University, Corneel Heymanslaan 10, 9000 Ghent, Belgium. ${ }^{2}$ Maccabi Healthcare Services and Sackler Faculty of Medicine, Tel Aviv University, Tel Aviv, Israel. ${ }^{3}$ College of Medicine \& Health Sciences, Sultan Qaboos University, Muscat, Oman. ${ }^{4}$ Sanofi-Aventis, Paris, France. ${ }^{5}$ Stat Process, Paris, France. ${ }^{6}$ Department of Cardiology, European Hospital Georges-Pompidou, Paris, France.
Received: 5 November 2019 Accepted: 12 March 2020 Published online: 07 April 2020

\section{References}

1. European Association for Cardiovascular Prevention \& Rehabilitation, Reiner Z, Catapano AL, De Backer G, Graham I, Taskinen MR, Wiklund O, Agewall S, Alegria E, Chapman MJ, et al. ESC/EAS guidelines for the management of dyslipidaemias: the task force for the management of dyslipidaemias of the European Society of Cardiology (ESC) and the European atherosclerosis society (EAS). Eur Heart J. 2011;32:1769-818.

2. Stone NJ, Robinson JG, Lichtenstein AH, Bairey Merz CN, Blum CB, Eckel RH, Goldberg AC, Gordon D, Levy D, Lloyd-Jones DM, et al. ACC/AHA guideline on the treatment of blood cholesterol to reduce atherosclerotic cardiovascular risk in adults. J Am Coll Cardiol. 2013;2014(63):2889-934.

3. Murphy A, Faria-Neto JR, Al-Rasadi K, Blom D, Catapano A, Cuevas A, LopezJimenez F, Perel P, Santos R, Sniderman A, et al. World heart federation cholesterol roadmap. Glob Heart. 2017;12:179-97 e175.

4. Reiner Z. Statins in the primary prevention of cardiovascular disease. Nat Rev Cardiol. 2013;10:453-64.

5. Keshishian A, Tan H, Xie L, Baser O. Examination of the economic burden of dyslipidemia in the veterans health administration population. Value Health. 2016;19:A46.

6. Fox KM, Wang L, Gandra SR, Quek RGW, Li L, Baser O. Clinical and economic burden associated with cardiovascular events among patients with hyperlipidemia: a retrospective cohort study. BMC Cardiovasc Disord. 2016; 16:13.

7. Hallberg S, Gandra SR, Fox KM, Mesterton J, Banefelt J, Johansson G, Levin $L A$, Sobocki P. Healthcare costs associated with cardiovascular events in patients with hyperlipidemia or prior cardiovascular events: estimates from Swedish population-based register data. Eur J Health Econ. 2016;17:591-601.

8. Smith DG. Epidemiology of dyslipidemia and economic burden on the healthcare system. Am J Manag Care. 2007;13(Suppl 3):S68-71.

9. Graham JH, Sanchez RJ, Saseen JJ, Mallya UG, Panaccio MP, Evans MA. Clinical and economic consequences of statin intolerance in the United States: results from an integrated health system. J Clin Lipidol. 2017;11:70-9 e71.

10. Zhao Y, Zabriski S, Bertram C. Associations between statin adherence level, health care costs, and utilization. J Manag Care Spec Pharm. 2014;20:703-13.

11. Kotseva K, De Bacquer D, Jennings C, Gyberg V, De Backer G, Ryden L, Amouyel P, Bruthans J, Cifkova R, Deckers JW, et al. Time trends in lifestyle, risk factor control, and use of evidence-based medications in patients with coronary heart disease in Europe: results from 3 EUROASPIRE surveys, 19992013. Glob Heart. 2017;12:315-22 e313.

12. Waters DD, Brotons C, Chiang CW, Ferrieres J, Foody J, Jukema JW, Santos $R D$, Verdejo J, Messig M, McPherson R, et al. Lipid treatment assessment project 2: a multinational survey to evaluate the proportion of patients achieving low-density lipoprotein cholesterol goals. Circulation. 2009;120: 28-34.

13. Reiner Z, De Backer G, Fras Z, Kotseva K, Tokgozoglu L, Wood D, De Bacquer $D$, Euroaspire investigators. Lipid lowering drug therapy in patients with coronary heart disease from 24 European countries--findings from the EUROASPIRE IV survey. Atherosclerosis. 2016;246:243-50.

14. Shioji K, Izuhara M, Mitsuoka H, Uegaito T, Matsuda M, Kishiwada Atherosclerosis Prevention Study G. Achievement rates of Japan Atherosclerosis Society Guidelines 2007 LDL-cholesterol goals with rosuvastatin or atorvastatin in patients who had not achieved their goal with atorvastatin. Cardiovasc Ther. 2014(32):97-104.

15. Chiang CE, Ferrieres J, Gotcheva NN, Raal FJ, Shehab A, Sung J, Henriksson KM, Hermans MP. Suboptimal control of lipid levels: results from 29 countries participating in the centralized pan-regional surveys on the Undertreatment of Hypercholesterolaemia (CEPHEUS). J Atheroscler Thromb. 2016;23:567-87.

16. Gitt AK, Lautsch D, Ferrieres J, Kastelein J, Drexel H, Horack M, Brudi P, Vanneste B, Bramlage P, Chazelle F, et al. Contemporary data on low-density lipoprotein cholesterol target value attainment and distance to target in a cohort of 57,885 statin-treated patients by country and region across the world. Data Brief. 2016;9:616-20.

17. Zhao M, Cooney MT, Klipstein-Grobusch K, Vaartjes I, De Bacquer D, De Sutter J, Reiner Z, Prescott E, Faggiano P, Vanuzzo D, et al. Simplifying the audit of risk factor recording and control: a report from an international study in 11 countries. Eur J Prev Cardiol. 2016;23:1202-10. 
18. Danchin N, Almahmeed W, Al-Rasadi K, Azuri J, Berrah A, Cuneo CA, Karpov Y, Kaul U, Kayikcioglu M, Mitchenko O, et al. Achievement of low-density lipoprotein cholesterol goals in 18 countries outside Western Europe: the international ChoLesterol management practice study (ICLPS). Eur J Prev Cardiol. 2018;25:1087-94.

19. Reiner Z, Catapano AL, De Backer G, Graham I, Taskinen MR, Wiklund O, Agewall S, Alegria E, Chapman MJ, Durrington P, et al. ESC/EAS guidelines for the management of dyslipidaemias. Eur Heart J. 2011;32:1769-818.

\section{Publisher's Note}

Springer Nature remains neutral with regard to jurisdictional claims in published maps and institutional affiliations.

Ready to submit your research? Choose BMC and benefit from:

- fast, convenient online submission

- thorough peer review by experienced researchers in your field

- rapid publication on acceptance

- support for research data, including large and complex data types

- gold Open Access which fosters wider collaboration and increased citations

- maximum visibility for your research: over $100 \mathrm{M}$ website views per year

At $\mathrm{BMC}$, research is always in progress.

Learn more biomedcentral.com/submissions 